\title{
Critical review of economic evaluation studies of interventions promoting low-fat diets
}

\author{
Giovanni Fattore, Francesca Ferrè, Michela Meregaglia, Elena Fattore, and Carlo Agostoni
}

Various national and local policies encouraging healthy eating have recently been proposed. The present review aims to summarize and critically assess nutrition-economic evaluation studies of direct (e.g., diet counseling) and indirect (e.g., food labeling) interventions aimed at improving dietary habits. A systematic literature review was performed by searching 5 databases (PubMed, Ovid Medline, EconLit, Agricola, and Embase) using a combination of diet-related (fat, diet, intake, nutrition) and economics-related (cost-effectiveness, cost-benefit, cost-utility, health economics, economic evaluation) key words. The search yielded 36 studies that varied in target population, study design, economic evaluation method, and health/economic outcome. In general, all provide limited experimental evidence and adopt the framework of economic evaluations in healthcare. Certain important aspects were not well considered: 1) the non-health-related effects of nutrition interventions on well-being; 2) the private nature of food expenditures; 3) the distributional effects on food expenditures across socioeconomic groups; and 4) the general economic implications (e.g., agrofoods, import/export) of such interventions. Overall, the methodology for the economic evaluation of nutrition interventions requires substantial improvement.

๑) 2014 International Life Sciences Institute

\section{INTRODUCTION}

Dietary trends across the world are of increasing concern, with rates of obesity and overweight rising to epidemic proportions. ${ }^{1,2}$ Unhealthy diet and physical inactivity, together with tobacco and alcohol use, are key risk factors that contribute to a large proportion of the world's disease burden. ${ }^{3}$ Diseases and health conditions linked to poor diet include cardiovascular diseases, diabetes, and cancers, which represent the major contributors to avoidable mortality.

In recent decades, there has been a significant change in dietary habits and physical activity levels worldwide as a result of industrialization, urbanization, economic development, and food market globalization. ${ }^{3}$ Parallel to these changes, overnutrition has increased to the extent that $35 \%$ of adults worldwide are overweight (body mass index [BMI] $\geq 25 \mathrm{~kg} / \mathrm{m}^{2}$ ) and $12 \%$ are obese (BMI $\geq 30 \mathrm{~kg} /$ $\left.\mathrm{m}^{2}\right){ }^{4}$ Many governments have considered or implemented measures to address the causes of obesity by embracing increasingly comprehensive strategies that involve communities and key stakeholders (e.g., mass media campaigns and school-based and worksite interventions). In these interventions, attention has been focused on dietary improvements through nutrition education, health promotion, and counseling of individuals at

Affiliations: G Fattore is with the Centre for Research on Health and Social Care Management (CERGAS) and the Department of Policy Analysis and Public Management, Bocconi University, Milan, Italy. F Ferrè is with the Centre for Research on Health and Social Care Management (CERGAS), Bocconi University, Milan, and Università degli Studi di Parma, Parma, Italy. M Meregaglia is with the Centre for Research on Health and Social Care Management (CERGAS), Bocconi University, Milan, Italy. E Fattore is with the Department of Environmental Health Sciences, IRCCS Istituto di Ricerche Farmacologiche"Mario Negri," Milan, Italy. C Agostoni is with the Department of Maternal and Paediatric Science, University of Milan, Fondazione IRCCS Cà Grande, Ospedale Maggiore Policlinico, Milan, Italy.

Correspondence: G Fattore, Department of Policy Analysis and Public Management, Bocconi University, Via Roentgen 1, Milan 20136, Italy. E-mail: giovanni.fattore@unibocconi.it. Phone: +39-338-7816605. Fax: +39-02-58362581.

Key words: cost effectiveness, diet intervention, economic evaluation, nutrition, public policies, review 
risk. These interventions are also promoted on the basis of economic reasons because the healthcare costs associated with diseases and conditions related to unhealthy nutrition are substantial; estimates for costs associated with obesity alone are $1 \%$ to $3 \%$ of total healthcare expenditures in most countries (5\% to $10 \%$ in the United States). ${ }^{2}$

Lifestyle changes related to diet may improve health outcomes and reduce healthcare costs associated with overweight and other disorders. The relationship between nutrition and health is well documented, and the impact of various nutrition changes on health status has been widely investigated and reported. However, evidence about the effectiveness of such interventions is frequently limited and typically involves little awareness about their economic implications (i.e., the cost-effectiveness impact). ${ }^{5-9}$

The increasing interest in policy actions aimed at improving people's diet suggests a crucial role for an economic analysis of nutrition interventions for clinical translational science decision-making. ${ }^{7,10}$ The economics of nutrition can be understood as a process of researching and characterizing health and economic outcomes following nutrition interventions and nutrition recommendations. ${ }^{11}$ Such an approach is developing as part of evidence-based health and economic research activities aimed at informing decision-makers about strategies to promote healthier and more sustainable lifestyles. Methods for the economic evaluation of healthcare interventions have been developed and refined in the last three decades ${ }^{12}$; however, these methods have been developed primarily to assess healthcare technologies, such as drugs, devices, and medical procedures. By comparison, economic evaluations of broader public health interventions are scarce, and the methods are not as well established. ${ }^{13,14}$ No specific reflections or methods have been developed for interventions targeting policy decisions in the area of nutrition, despite the clear need and important stakes, which include industrial interests and direct consumer protection. ${ }^{10}$

Although the benefits of nutritional interventions are potentially enormous, the extant economic literature offering a full assessment of these interventions appears limited. A recent review by Gyles et al. ${ }^{9}$ identified 30 studies presenting economic analyses of dietary improvements. These studies cover different types of interventions and are based on a variety of methods to generate data and to provide economic summaries. They generally suggest that interventions are either cost saving (i.e., they improve health outcomes while reducing healthcare expenditures) or cost effective (i.e., they improve health outcomes at acceptable levels of additional costs). Although the review corroborates the claim that nutritional interventions may be highly desirable, it also high- lights the variations in the methods used and the lack of common direction about how to conduct economic studies in this area.

The aim of the present study is to summarize and critically assess economic evaluation studies conducted on direct (e.g., counseling) or indirect (e.g., food labeling) interventions aimed at promoting voluntary dietary improvements through reduction of fat intake. Indeed, it is widely reported in the nutrition literature that dietary fat intake plays a role in the development of obesity, since reducing the amount of fat intake decreases the gap between total energy intake and total energy expenditure and thus can be suggested as an effective strategy for limiting the current epidemic of obesity. ${ }^{15}$ This review examined studies that adopted a cost-benefit type of analysis, i.e., they produced information about the positive (improved health and/or reduced costs) and negative (any adverse consequence on well-being and/or additional costs) effects of nutrition interventions whose main aim was improving dietary habits via the reduction of fat intake in diets. It is expected that such evaluations are strongly influenced by those developed in the field of economic evaluation of healthcare programs and health technology assessment. ${ }^{12,16} \mathrm{~A}$ number of nutrition interventions that are expected to motivate individuals to modify their nutrition behavior have been considered, including nutritional counseling, information campaigns, food labeling, and extensive educational efforts. The primary aim of such interventions is reducing the intake of fats and other fattening nutrients (e.g., salt, carbohydrates), or promoting the consumption of healthy nutrients (e.g., vegetables and fruits) to rebalance dietary regimens.

\section{METHODS}

A systematic literature review was performed by following the PRISMA statement (Preferred Reporting Items for Systematic Reviews and Meta-Analyses) ${ }^{17}$ and searching 5 bibliographic databases (PubMed, Ovid MEDLINE, EconLit, Agricola, and Embase). The search strategy was executed using a combination of two sets of keywords: 1) diet-related keywords: fat, diet, intake, nutrition; and 2) economics-related terms: cost-effectiveness, cost-benefit, cost-utility, health economics, economic evaluation. The search was performed on titles and abstracts only. The search was filtered for English full-text papers only and included literature published up to March 31, 2013. All database search results were imported into EndNote software (version 6; Thomson Reuters, Philadelphia, PA) to identify duplicate papers and to screen titles and abstracts.

Two independent authors (FF and MM) investigated the relevance of the papers obtained from the initial 
computerized search by screening the titles and abstracts. The full text of the published literature was obtained if either reviewer identified a citation as potentially relevant. In the second phase of screening, both reviewers independently reviewed the full-text version of all included articles. Any discrepancies between reviewers were discussed until a consensus was reached. Follow-up searches (manual search) were conducted on citations found in eligible studies.

Records were screened for inclusion on the basis of predefined criteria. The papers were considered for inclusion if the following criteria were met: 1 ) they addressed interventions that stimulated voluntary changes in dietary habits at the individual or group level, either directly (e.g., diet counseling) or indirectly (e.g., food labeling); 2) they reported an economic evaluation of any type that documented clinical or other outcomes to improve well-being and information about the use of scarce resources (costs); and 3) they were original studies (i.e., no review articles, meeting abstracts, or editorials). Because the review focused on intentional dietary interventions, studies addressing clinical (i.e., enteral or parenteral) nutrition or undernutrition were excluded. At the same time, papers concerning fiscal policies (i.e., taxes) or other prescriptive measures (e.g., legislation or bans) were excluded unless they compared such policies with other interventions that were voluntary in nature. Studies addressing interventions of industrial modification (e.g., low-fat margarine) or fortification (e.g., with folic acid) of foods were also excluded. A predeveloped data abstraction form was used to extract information regarding title, authors, publication journal, country, year, policy instrument, target population, sample size, study design, time horizon, intervention action, comparator, clinical and economic outcomes, cost assessment, study perspective, statistical methods, data sources, and study results. Studies were assessed using the standard checklist for critical appraisal of economic evaluation studies, ${ }^{12}$ complemented by the Consensus on Health Economic Criteria CHEC-list that focuses on the quality of economic evaluations. ${ }^{18}$

\section{RESULTS}

The literature search yielded 665 potentially relevant papers with 326 duplicates that were removed, leaving 339 papers to be screened by title and abstract. After exclusion and manual searches, 36 relevant studies were identified for the review. ${ }^{19-54}$ Figure 1 outlines the flow of information through the different phases of the review and highlights the reasons for exclusion. The included studies were mainly published in US and UK journals; 3 articles were published between 1992 and 2001, 13 between 2002 and 2007, and 20 between 2008 and 2013.

\section{Study design and population}

Studies were based on data from the United States $(n=16)$, countries in Europe ( $n=11 ; 5$ from the Netherlands and 6 from other countries), Australia $(n=7)$, Vietnam $(\mathrm{n}=1)$, and Canada $(\mathrm{n}=1)$ (Table 1$)$.

A variety of study designs were used. Almost half of the studies $(n=16)$ were based on models, ${ }^{20,24,26,27,29,30,33-35,38-41,46,48,51} 7$ were based on data generated through full experiments, ${ }^{22,31,44,47,50,52,53} 3^{23,25,54}$ were classified as quasi-experiments (i.e., with nonequivalent control groups), $3^{37,43,46}$ were based on observational data with no control groups, and $7^{19,21,28,32,36,42,49}$ adopted a mix of approaches (which typically involved modeling on the basis of trial results).

Among the modeling studies, the Markov chain was the preferred approach $(n=9)$ and was generally used to simulate chronic disease progression. ${ }^{19-21,28,30,32,39,45,48}$ Studies adopting an experimental design (either alone or complemented by modeling) included randomized controlled trials $(\mathrm{n}=8)$, for which the units of randomization were individuals, $19,21,22,28,36,47,50,52$ and cluster randomized controlled trials $(n=5)$, for which the units of randomization were groups of individuals (i.e., health centers, schools, and worksites $\left.{ }^{31,32,44,49,53}\right)$. The nonexperimental studies included 1 prospective ${ }^{37}$ and 2 retrospective cohort studies. ${ }^{43,46}$ Half of the included studies selected study participants on the basis of a single criterion (e.g., $\mathrm{BMI}$ ), whereas the other half combined 2 or more inclusion criteria (e.g., age, BMI, and the presence of other clinical conditions). In 21 studies, interventions were targeted to healthy individuals (i.e., the general population), whereas the remaining 15 studies ${ }^{19,21,22,25,28,30,32,36,40,41,47,50-53}$ selected participants with specific medical conditions (mainly overweight and obesity, diabetes, hypertension).

When examining the sample size by study design (excluding models), important differences were observed. Quasi-experimental studies, such as Wootan et al., ${ }^{54}$ included the highest number of individuals $(n=35,000)$. In studies based on a pure experimental design (i.e., without modeling), the average number of individuals was 2,113 (range: $129-10,144),{ }^{31,50}$ with cluster randomized controlled trials recruiting more participants than randomized controlled trials. Nonexperimental studies enrolled from 368 to 3,100 subjects, ${ }^{43,46}$ whereas the mixed designs enrolled between 64 and 3,234 individuals. ${ }^{19,36}$

\section{Characteristics of nutritional interventions, clinical outcomes, and comparators}

Studies were classified according to the categories of foods and/or nutrients addressed by the nutrition interventions. According to the main objective of this review, in 10 studies $^{21,22,30-33,39-41,54}$ the intervention consisted 


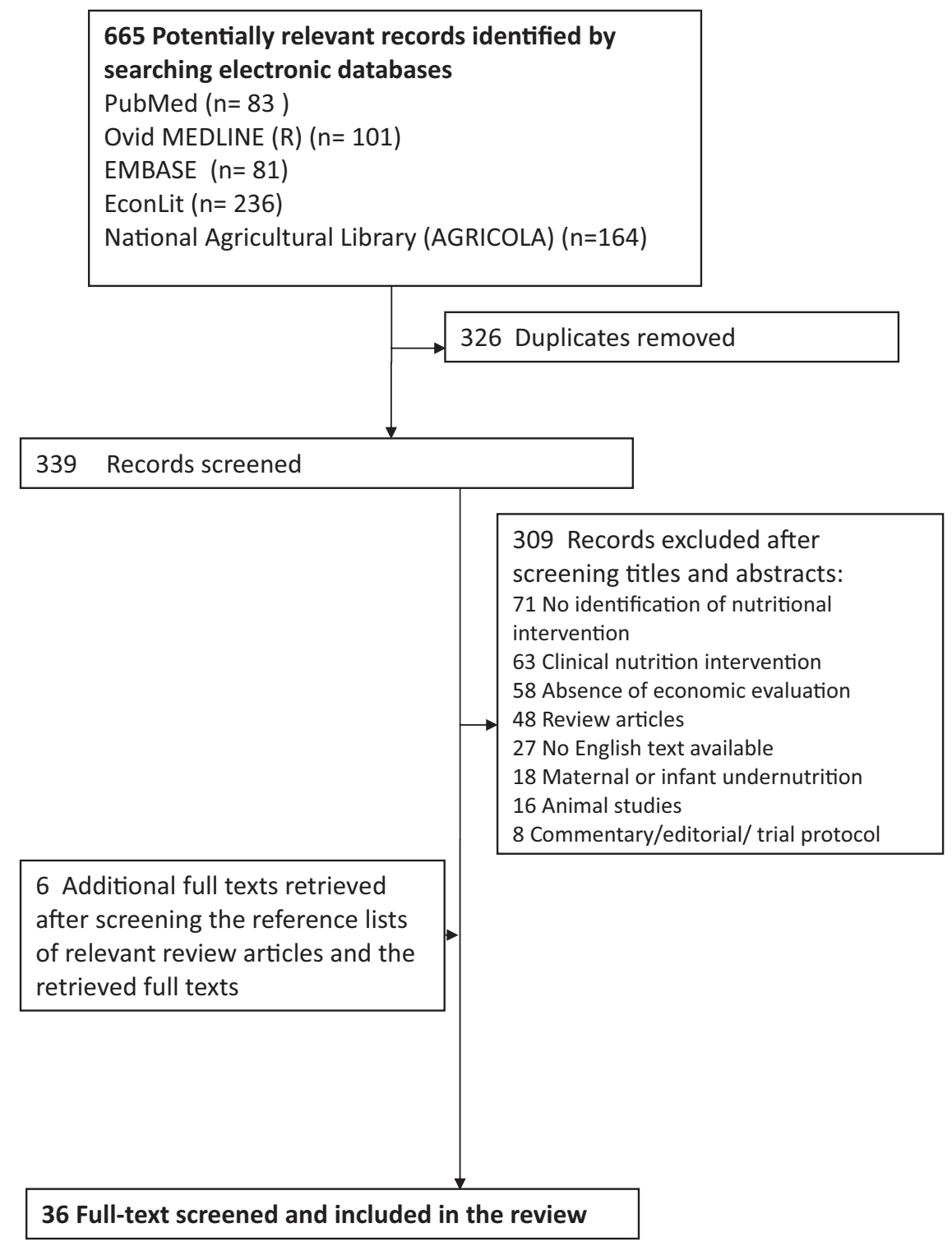

Figure 1 Flow chart of the literature screening process.

specifically of promoting a low-fat diet or a replacement of saturated fats with polyunsaturated fats in diet; the other studies involved encouraging salt intake reductions $(\mathrm{n}=5),{ }^{20,24,35,36,48}$ fruit and vegetable consumption $(\mathrm{n}=5),{ }^{27,34,44,49,52}$ low-calorie diets $(\mathrm{n}=3)^{26,38,51}$ lowcarbohydrate diets $(\mathrm{n}=1),{ }^{50}$ and so-called "junk food" (i.e., pastries, sausages, pre-made meals) avoidance $(\mathrm{n}=1){ }^{45}$ Two studies ${ }^{28,42}$ assessed the benefits of a Mediterranean diet (i.e., high monounsaturated-to-saturated fat ratio) versus more Westernized diets. Nine studies ${ }^{19,23,25,29,37,43,46,47,53}$ reported evaluations of unspecified or generic dietary improvements. Three studies $32,39,40$ combined 2 different primary interventions (e.g., low-fat diet and salt reduction). In the reviewed studies, diet interventions were frequently associated with secondary interventions targeting other health-related behaviors $(\mathrm{n}=10)$, especially physical activity and, sometimes, also smoking and alcohol intake reduction. In these studies, ${ }^{19,20,30,32,36,40,44,47,52,53}$ it may be difficult to attribute a health improvement to each specific lifestyle change investigated.

Nutritional interventions were also classified on the basis of their health-related expected outcome and the policy instrument adopted to promote such behavioral change. As the first inclusion criterion was the voluntary nature of the nutritional intervention, the instrument preferred by the retrieved studies was nutritional counseling $(n=24)$ : in these interventions, individuals were educated about a healthier diet and lifestyle through traditional individual and/or group lessons with a dietician or through innovative instruments such as video-lesson packets, picture books, home visits, phone discussions, 


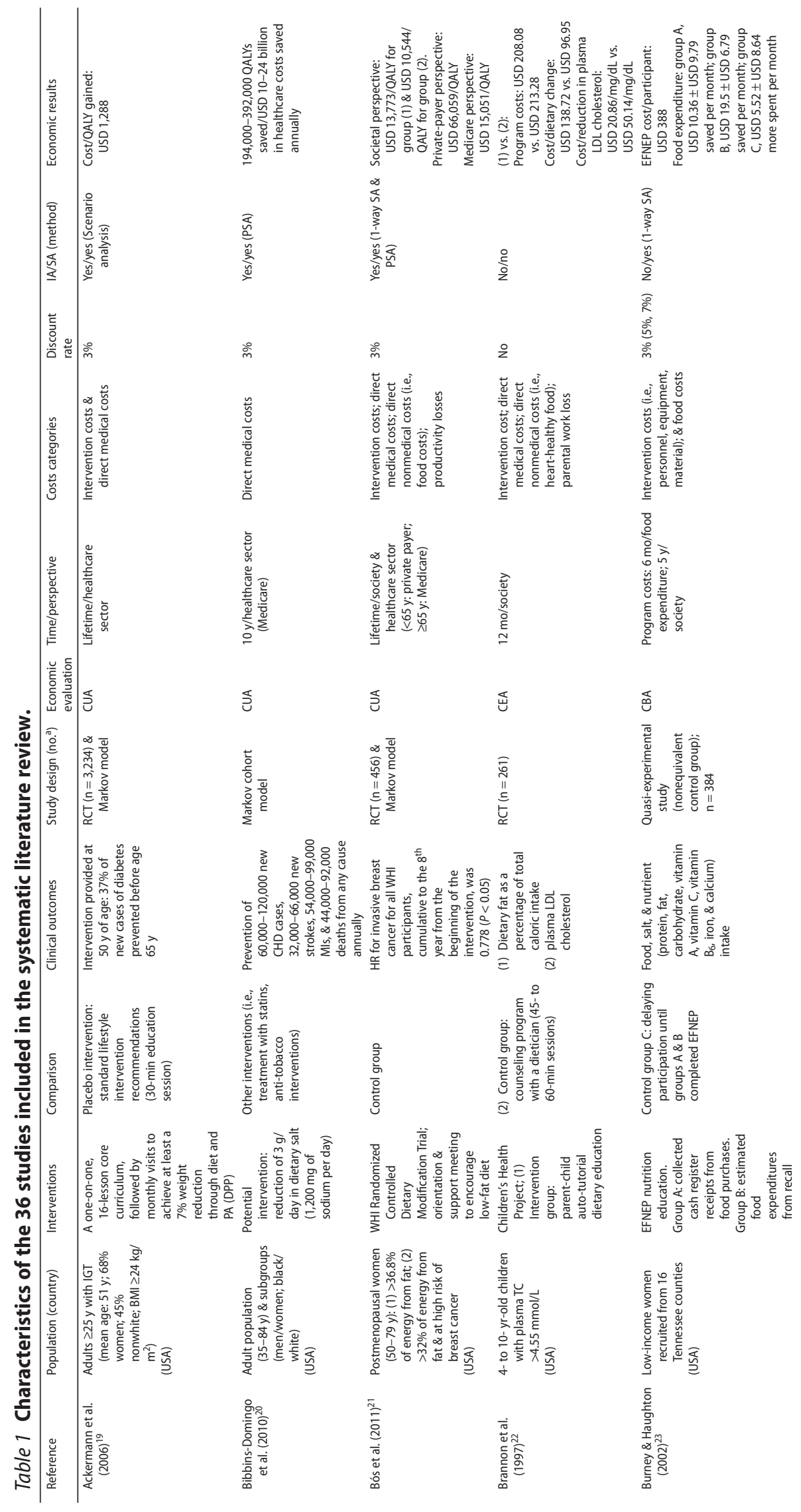




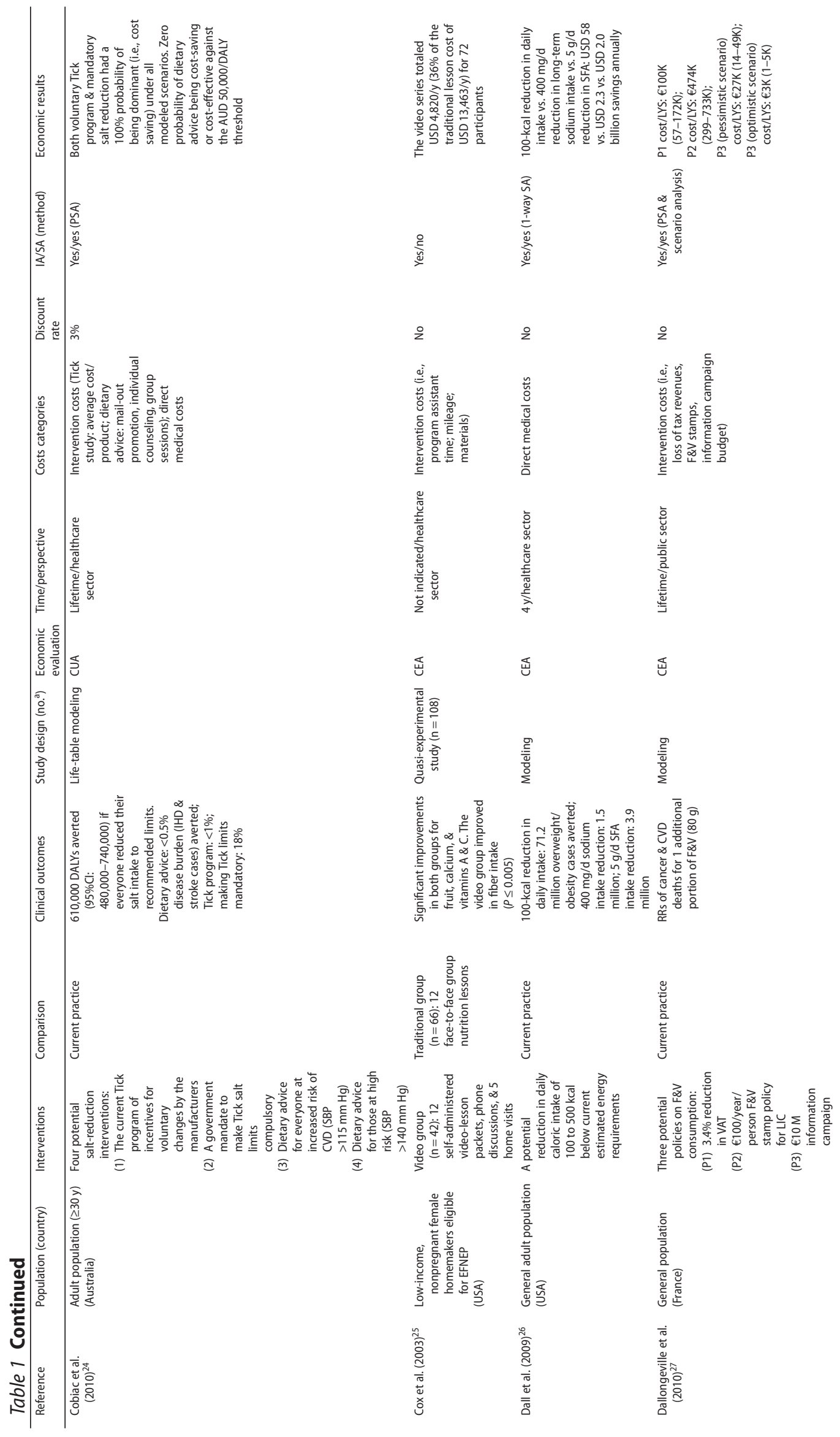



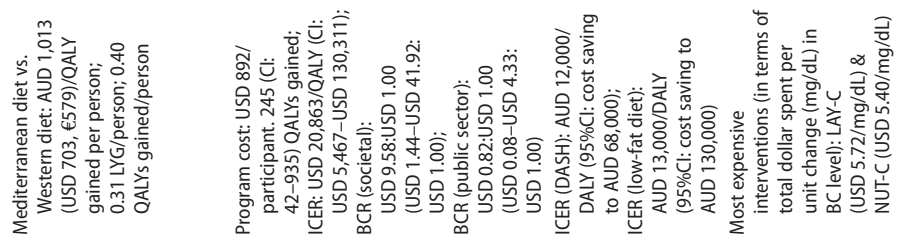

㐫

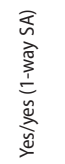

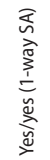

$\stackrel{\circ}{\stackrel{2}{2}}$

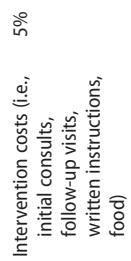

ํํ
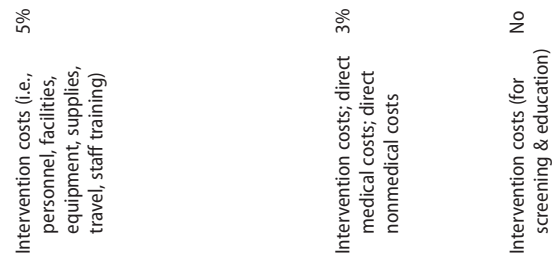

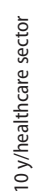
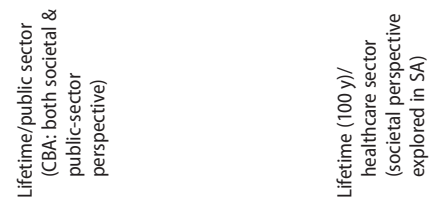

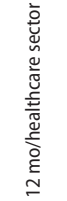

焉

ญ̊

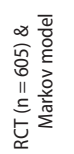

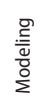

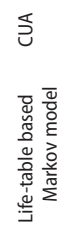

出
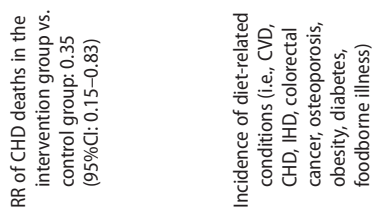

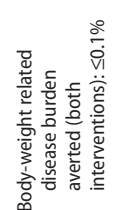

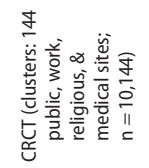

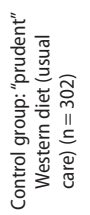
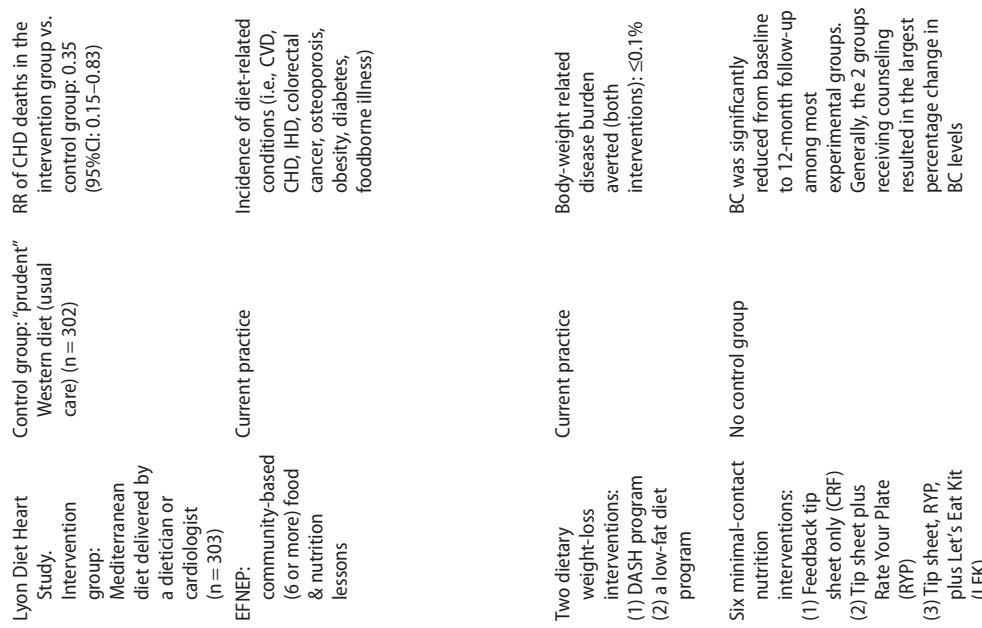

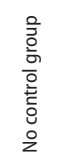
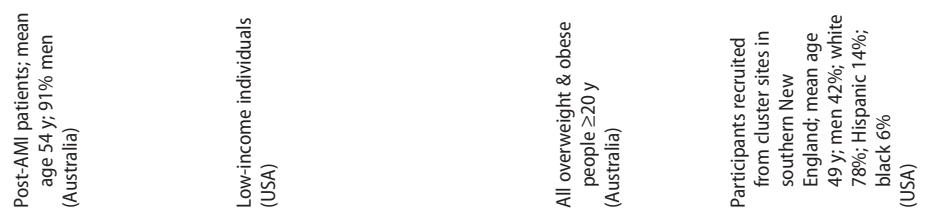

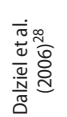

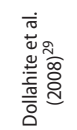

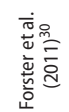

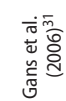




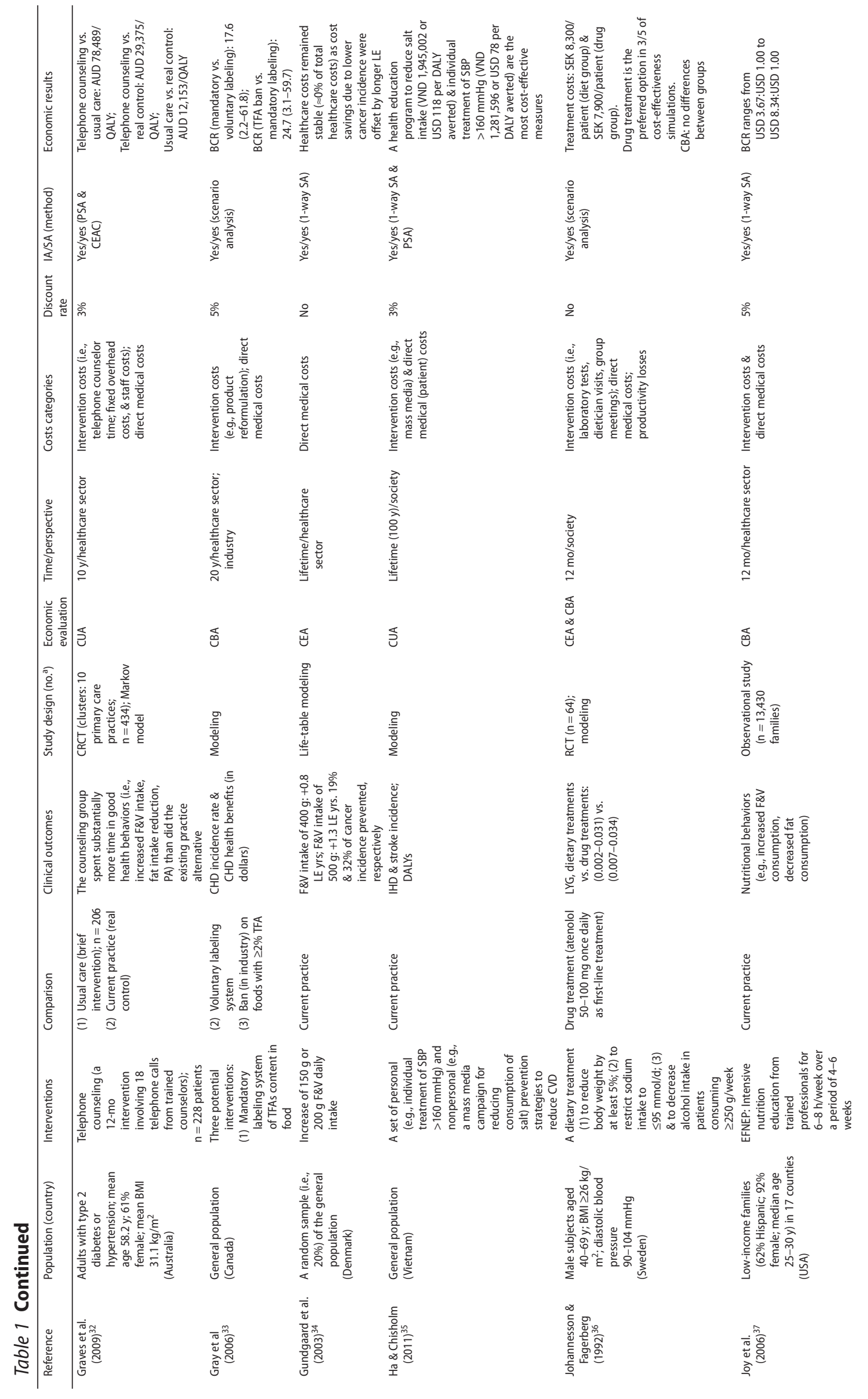



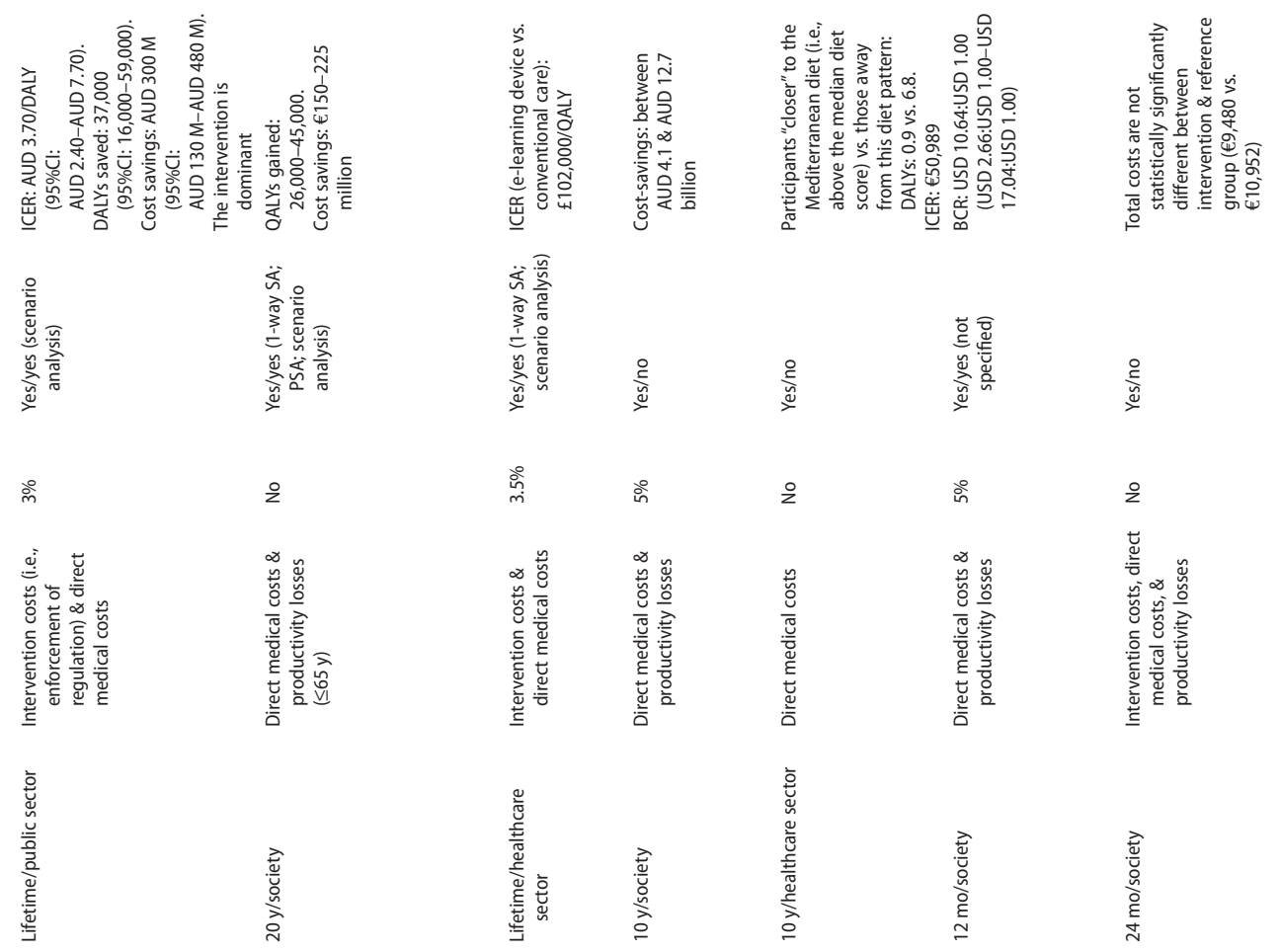

111

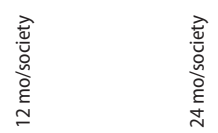

孚

尌

焉昏

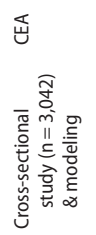

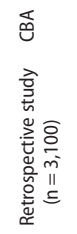

巴巴

I

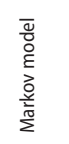

量

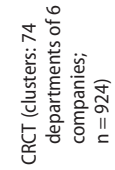

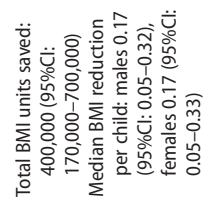
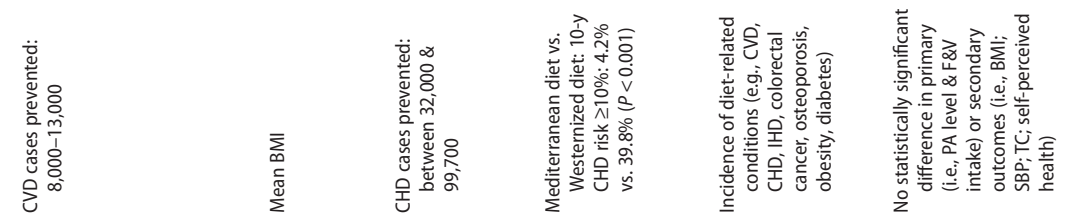

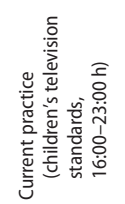

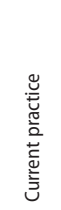

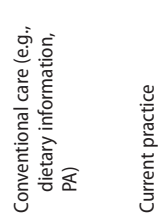
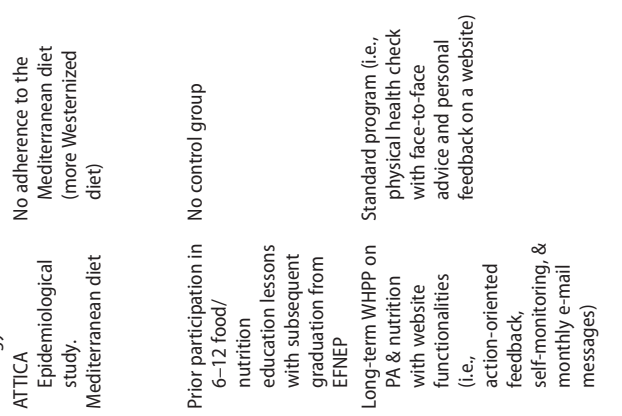

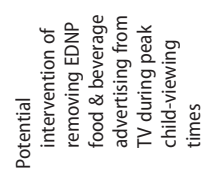
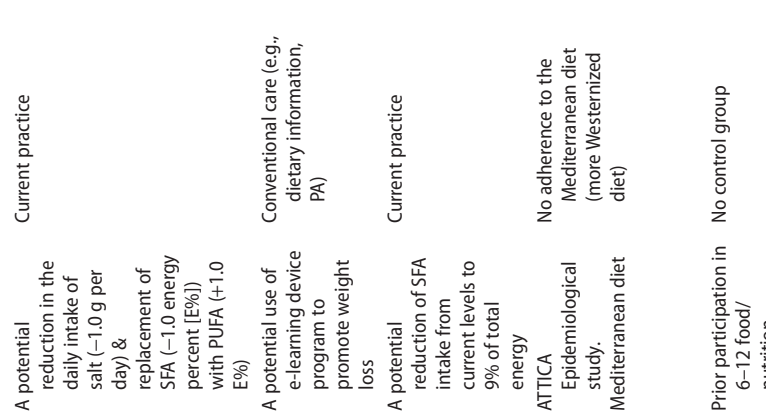

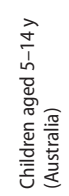

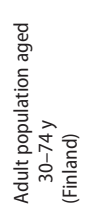

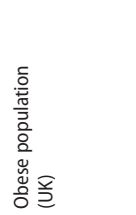

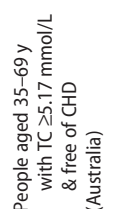

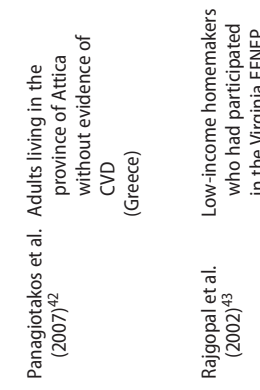

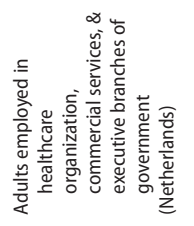

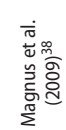

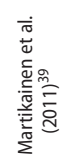

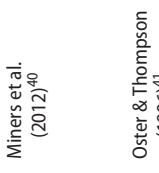

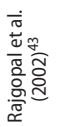

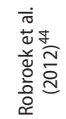




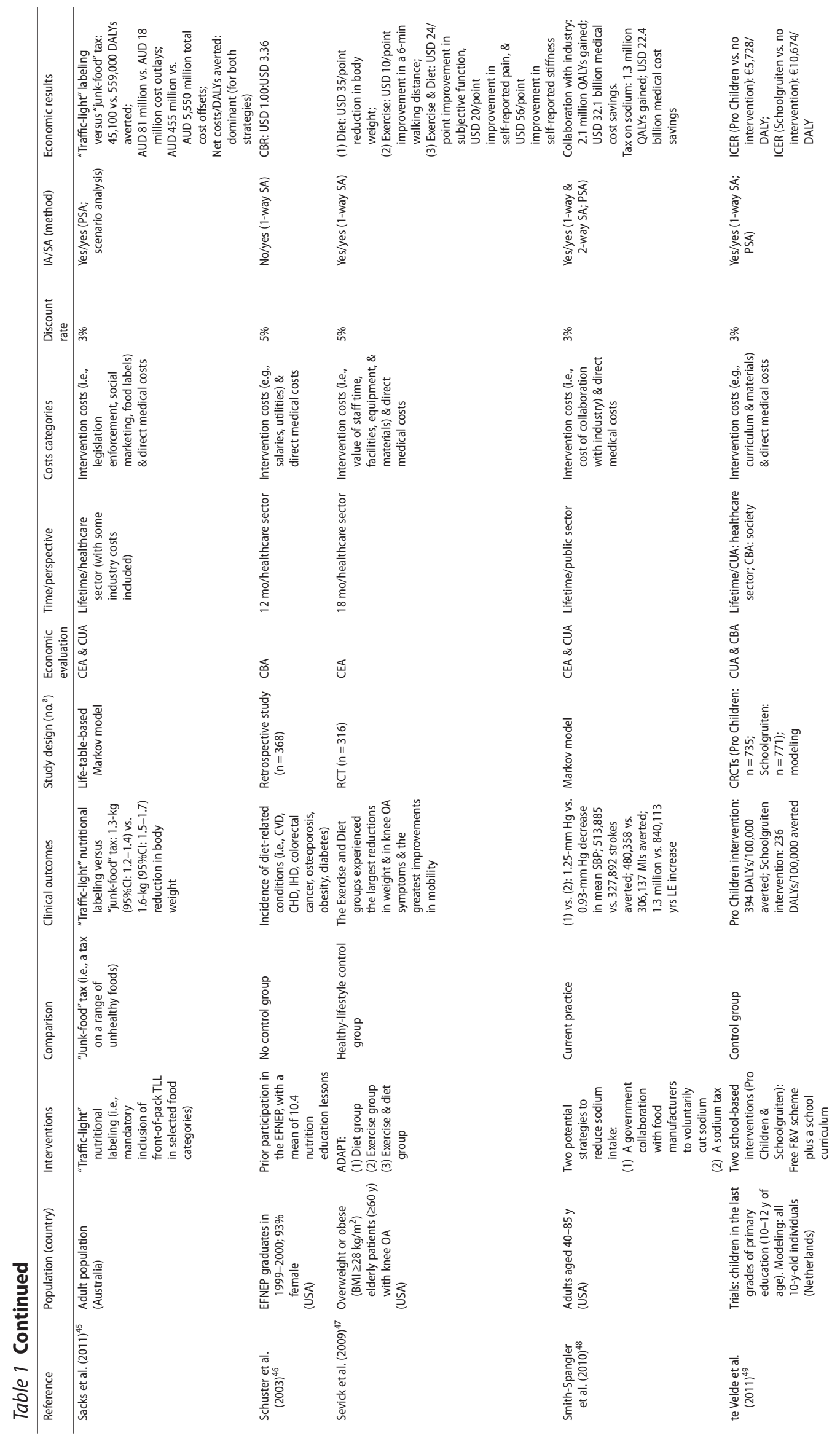



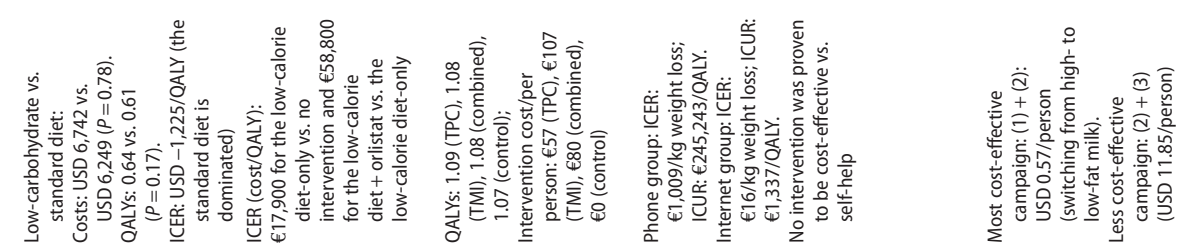

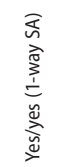
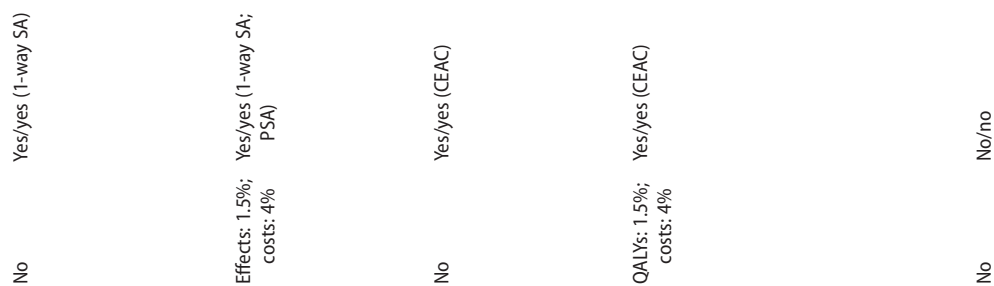

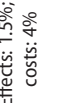

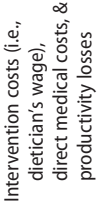

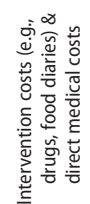

2 के

2

옹

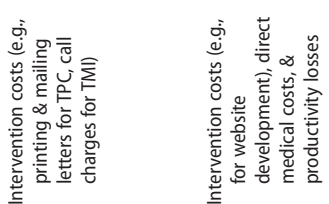

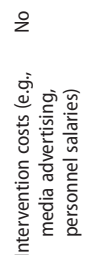

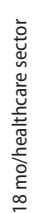

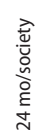

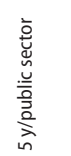

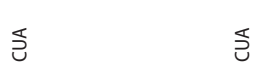

矛

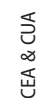

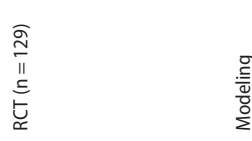

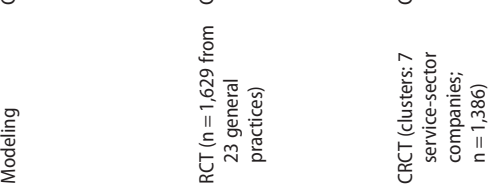
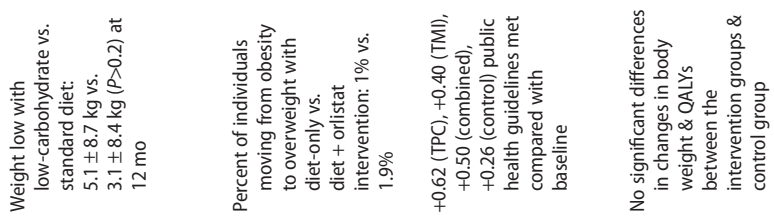

畓

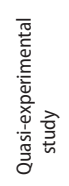

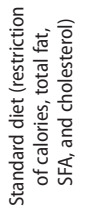

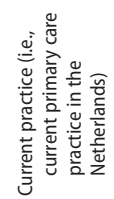

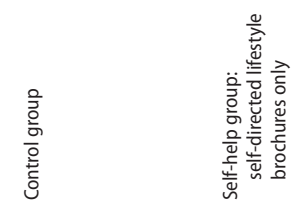

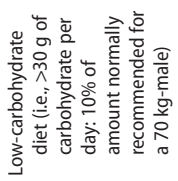
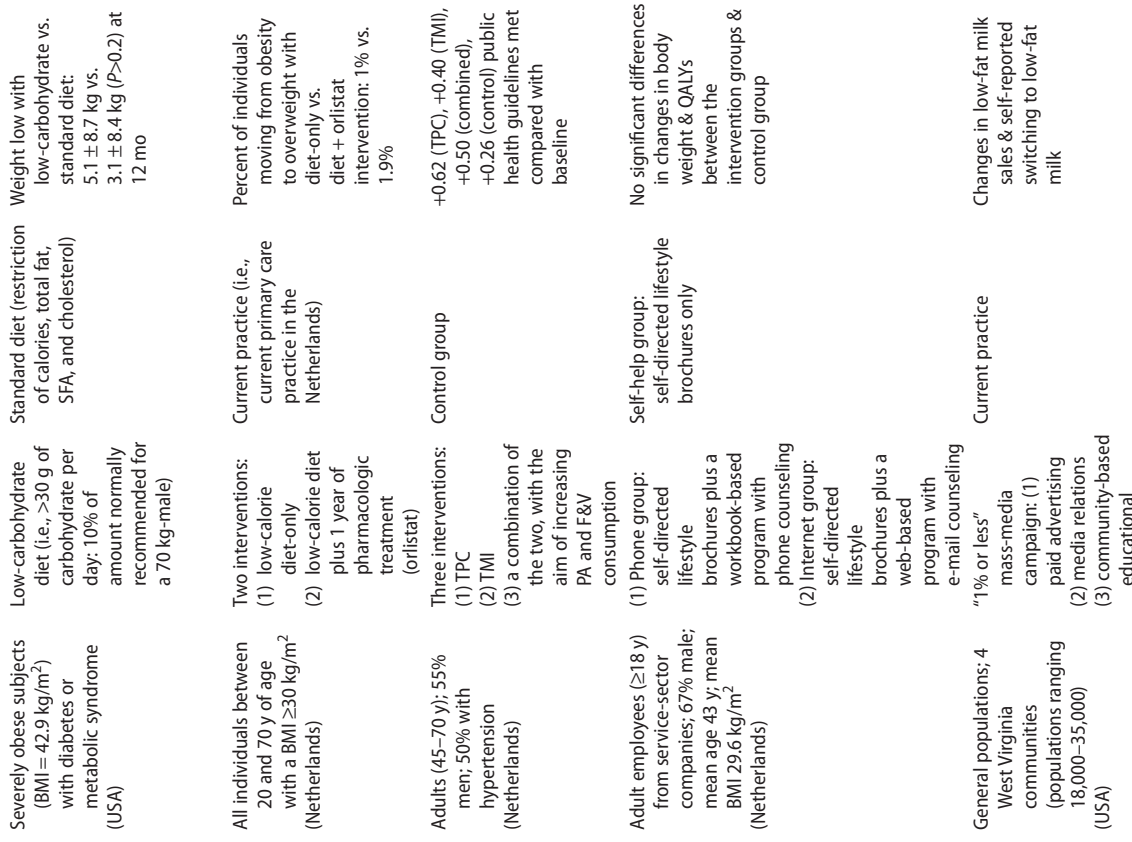

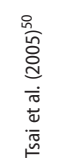

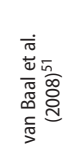

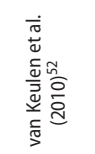

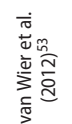

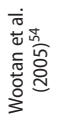

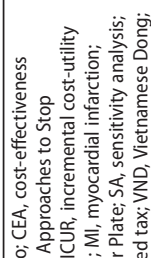

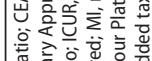

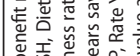

잉

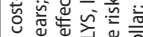

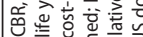

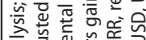

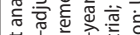

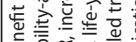

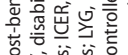

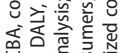

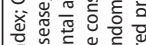

든

管

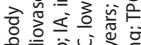

部을

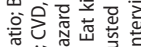

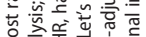

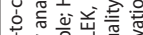

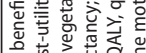

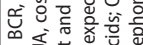

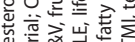

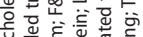

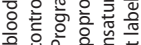

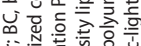

क्ष

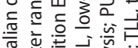

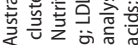

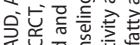

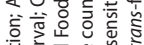

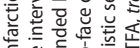

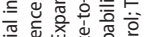

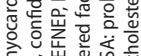

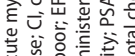

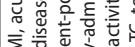

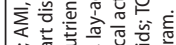

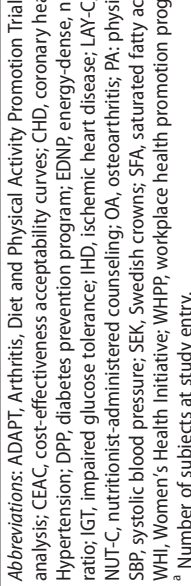


and e-mail messages. In 3 articles, ${ }^{35,38,54}$ the nutrition education was carried out at a population level; among them, Wootan et al. ${ }^{54}$ assessed the effects of a public information campaign encouraging a switch from whole to skim milk, whereas Magnus et al. ${ }^{38}$ evaluated a control program of energy-dense food and beverage TV advertising. Four modeling studies ${ }^{27,33,45,48}$ predicted the health-related and economic effects of potential policy interventions, such as banning industrial trans-fatty acids or raising taxes on a range of unhealthy foods. According to the purpose of this review, these prescriptive measures were compared with voluntary nutrition interventions (e.g., public information campaign, food labeling). In the remaining models, ${ }^{20,26,34,39,41}$ the nutrition intervention was not specified, as the objective was to predict the effects of potential diet improvements (e.g., a potential reduction of saturated fatty acids intake) without reporting how to get them.

Twenty-seven studies targeted specific medical conditions, mainly cardiovascular disease (i.e., stroke, myocardial infarction; $\mathrm{n}=10)^{20,24,27,28,33,35,39,41,42,48}$ and overweight and obesity $(\mathrm{n}=10)^{26,30,36,38,40,45,47,50,51,53} ; 2$ papers focused on cancer ${ }^{21,34}$ and 2 on diabetes, ${ }^{19,32}$ and 3 addressed outcomes associated with a mix of diet-related diseases such as cardiovascular disease, cancer, diabetes, and osteoporosis. ${ }^{29,43,46}$ Nine studies did not address any specific health-related condition and generically aimed to improve food-related behaviors. $22,23,25,31,37,44,49,52,54$

In most studies $(n=21)$, nutritional interventions were compared with no intervention. Six studies ${ }^{22,25,28,33,42,45}$ compared 2 alternative nutritional programs, with the intervention group typically testing a more innovative approach to dietary behavioral change than the control group (e.g., video-lesson packets vs. faceto-face group nutrition lessons; Cox et al..$^{25}$ ). Seven studies ${ }^{19,23,32,44,47,50,53}$ used the same primary intervention as a comparator, but in a milder version (e.g., a 12-month intervention vs. a brief intervention; Graves et al. ${ }^{32}$ ). In 2 studies, ${ }^{20,36}$ the alternative action was a non-nutritional intervention (i.e., a drug treatment with atenolol or statins).

\section{Characteristics of economic evaluations}

Three types of economic evaluations developed to assess health technologies were used in the reviewed studies: cost-effectiveness analysis $(n=11)$, which presents economic results as cost per unit of health gain (e.g., cost per case averted or cost per year of life gained); cost-utility analysis $(n=13)$, in which the unit of benefit is expressed in terms of an index capturing both quantity and quality of life, which are typically expressed as quality-adjusted life years (QALYs) or disability-adjusted life years (DALYs); and cost-benefit analysis, in which both costs and benefits are expressed in monetary terms $(n=5)$. Seven studies used a combined design of costeffectiveness analysis, cost-benefit analysis, and/or costutility analysis. There was substantial variation in the types of costs used to monetize the use of resources, with most studies $(n=29)$ reporting direct costs associated with the implementation of the nutritional intervention (e.g., dietician salary). When the policy instrument adopted was a public information campaign, the intervention costs included media and/or communication costs (e.g., media advertising; Wootan et al..$^{54}$ ). Modeling studies predicting the impact of legal (or fiscal) measures generally estimated the costs of collaboration between government and industry, the costs of enforcing legislation or administering taxes, and, in one case, ${ }^{33}$ the industrial costs of product reformulation. On the contrary, 7 studies did not include any intervention costs because the intervention action was not specified, as was often the case in modeling studies. ${ }^{20,26,34,39,41-43}$

Direct medical costs (e.g., hospitalization, emergency room, prescription medications, and medical consultations) associated with the treatment of the targeted dietrelated medical conditions were included in the vast majority of the studies $(n=26)$. Three studies also estimated direct nonmedical costs, such as healthier food costs and travel costs to healthcare facilities. ${ }^{22,23,30}$ Eight studies incorporated productivity losses, foregone wages, and time used by patients and family caregivers to obtain treatments. ${ }^{22,36,39,41,43,44,50,53} \mathrm{~A}$ discount factor ranging from $3 \%$ to $7 \%$ was applied to future costs and effects in 23 studies.

On the basis of the cost categories included in their analyses, papers were classified according to 3 perspectives. Twenty studies adopted a narrow perspective in which only costs borne by the healthcare system were included. Thirteen studies included other costs to proxy a societal perspective; typically, these costs refer to nonmedical services (e.g., social care), productivity losses, and time spent by patients and caregivers. $^{21-23,30,35,36,39,41,43,44,49,50,53}$ Three studies ${ }^{27,29,54}$ were classified according to a public-sector perspective because they included only costs supported by governmental bodies to implement the nutrition intervention (e.g., media advertising, information campaigns, fruit and vegetable stamps for low-income consumers), with no reference to the impact on healthcare expenditure. The level of specification of cost analysis and the approach used for cost measurement differ widely across studies. Four studies ${ }^{25,28,31,52}$ that adopted a healthcare-sector perspective limited their analysis to the intervention costs (e.g., for screening and education) without assessing disease-related costs. By contrast, Gray et al. ${ }^{33}$ and Sacks et al. ${ }^{45}$ added certain industry costs to the healthcare costs. Two US-based studies ${ }^{20,21}$ distinguished between 
public and private payers. However, all other studies ignored the distinction between public and private sources of costs induced by the intervention and did not discuss their implications in equity terms.

The length of follow-up of the studies with a longitudinal design ranged from 6 months ${ }^{23}$ to 10 years. ${ }^{28,32}$ Pure experimental studies (i.e., without modeling) typically had a limited length of follow-up (i.e., 2 years maximum ${ }^{44,53}$ ), which, in other papers, was extended through modeling techniques. Modeling studies had the longest time horizon because they generally predicted health and economic outcomes over the individuals' entire lifespans. None of the studies provided direct empirical evidence of the longterm effects of interventions.

The vast majority of the studies $(n=29)$ tested the robustness of the results through a sensitivity analysis. The techniques most frequently adopted were one-way sensitivity analysis $(\mathrm{n}=17)$ and probabilistic sensitivity analysis (i.e., Monte Carlo simulations; $\mathrm{n}=11$ ). Some studies also presented their results by utilizing different scenarios (i.e., scenario analysis; $\mathrm{n}=8$ ), while a few others $(n=3)$ used cost-effectiveness acceptability curves. A considerable number of authors $(n=10)$ adopted 2 or 3 sensitivity analysis techniques within the same study. ${ }^{21,27,32,35,39,40,45,48,49,51}$ The parameters that were tested primarily through the sensitivity analyses were timerelated variables (e.g., time horizon, discount rate, proportion of lifelong effects), disease incidence rates (e.g., cardiovascular risk), and direct medical costs.

\section{Study results/outcomes}

The main results of the economic evaluations were abstracted from each study (Table 1). To assess the consequences of a nutritional intervention, 21 studies adopted a clinical endpoint (e.g., strokes prevented), whereas 9 studies $^{22,31,38,40,44,45,47,50,53}$ used a clinical surrogate (biomarker), such as systolic blood pressure, highdensity lipoprotein, blood cholesterol, body weight, and BMI. Six studies ${ }^{23,25,32,37,52,54}$ limited their outcome assessment to the direct behavioral consequences of the intervention (e.g., intake of fat, fruit and vegetables, salt, and other nutrients; milk consumption, health guidelines met). The diet or nutritional status of the individuals participating in the studies was assessed through dietary questionnaires (e.g., diet diaries, 24-h diet recalls, food frequency questionnaires) or through health screening that measured biomarkers (e.g., blood cholesterol) of nutrient intake.

Different economic evaluation techniques were used to assess the interventions. The cost-utility analysis studies generally used QALY as a measure of well-being $(\mathrm{n}=13)$, although 7 studies measured improvement in terms of DALYs, ${ }^{24,30,35,38,42,45,49}$ the measure that corrects life expectancy for disability and that is used by the World Health Organization for assessing healthcare systems. ${ }^{55}$ Cost-effectiveness studies presented results in terms of cost per unit of physical measure of benefit, such as kcal, body weight, or total cholesterol. However, in these analyses, interventions were frequently cost saving, which made the issue of how to measure clinical benefits less relevant. In only 7 studies, ${ }^{23,29,33,36,43,46,49}$ the authors attempted to express both costs and benefits in monetary terms. In these studies, a variety of methodological choices were used, including the manner of presenting results (net monetary benefits, benefit-cost ratio, or [the opposite] cost-benefit ratio). Burney and Haughton ${ }^{23}$ derived the net present value to evaluate benefits and costs in a time dimension and expressed benefits as the difference between participants' food expenditures before and after the intervention. In other articles, ${ }^{33,43,46}$ benefits were characterized as disease costs avoided because of program results. In 2 recent studies, benefits were expressed as the value of QALYs gained ${ }^{29}$ and DALYs averted. ${ }^{49}$ Three studies ${ }^{29,36,49}$ adopted a willingness-topay approach to estimate the program benefits.

The vast majority of the studies concluded that the intervention examined was cost saving $(\mathrm{n}=18)^{20,22-26,29,33,37-39,41-43,45,46,48,49}$ or cost effective $(\mathrm{n}=11)^{19,21,27,28,31,32,34,35,47,51,54}$ compared with the status quo. By contrast, 7 studies ${ }^{30,36,40,44,50,52,53}$ concluded that the nutrition intervention was not cost effective or that health outcome improvements were negligible.

According to the Consensus on Health Economic Criteria CHEC-list, ethical and distributional issues should be considered when evaluating healthcare interventions. In the context of this review, individual accessibility and the affordability of nutritional programs are crucial elements to be analyzed. Fifteen papers mentioned some equity aspects: subgroup analyses (by race, gender, age, income, education, marital status, and health condition) were conducted in 5 studies, ${ }^{20,27,41,44,45}$ and 10 studies focused on individuals belonging to ethnic minority groups (e.g., Alaskan) or lower socioeconomic communities. ${ }^{21,23,25,29,31,32,38,43,46,50}$ However, no study discussed the equity implications of interventions that may impose additional private expenditure.

\section{DISCUSSION}

The research strategy used in this review identified only 36 published studies in the English language that performed economic evaluations of interventions aimed at improving nutritional habits. Given the potential health gains related to such interventions, the paucity of such studies is alarming and indicates that additional evidence in this area is needed. It is difficult to design evidencebased policies with so little empirical evidence. 
Fortunately, many more studies than those reviewed here have investigated nutritional interventions from only a clinical perspective. A systematic review of 33 randomized controlled trials and 10 cohort studies by Hooper et al. ${ }^{56}$ suggested that diets lower in total fat were associated with lower relative body weight $(-1.6 \mathrm{~kg}$; $95 \% \mathrm{CI}:-2 \mathrm{~kg}$ to $-1.2 \mathrm{~kg}$ ). The authors included studies in which a low-fat diet was compared with a normal diet or in which a low-fat diet plus any nondietary intervention was compared with a normal diet plus an identical nondietary intervention. However, the authors excluded studies in which a low-fat diet plus any nondietary intervention was compared with the normal diet alone.

Nutritional interventions have many socioeconomic implications, and it is difficult to conceive of evaluations that do not attempt to include these aspects. Without valid and reliable assessments of the effects on well-being and resource consumption attributable to interventions aimed at changing human habits, the risk of policy failure in conception and implementation is very real. To obtain adequate inputs from applied science, policymakers must know the health benefits of these interventions over both the short and the long term, the possible adverse effects (e.g., in terms of loss of well-being due to changes in nutrition), and the costs to individuals and public sector agencies. Without such evidence, policy can be only tentative and is subject to being easily overtaken by vested interests or biased perspectives. Although no specific guidelines or methodological recommendations for economic studies of nutritional interventions could be found in the current literature, some characteristics of the reviewed studies can be discussed. Modeling is the dominant evaluation strategy. As with the evaluation of drugs and other health technologies, it is virtually impossible to produce economic evidence without some modeling. However, to avoid simple speculations, models should be populated with data obtained from valid empirical studies and should follow good-practice recommendations. It is outside the scope of this study to reflect upon the type of empirical designs that are best suited to provide economic evidence for nutritional policies. Such reflections should consider the pros and cons of experiments, quasi-experiments, and observational studies and, equally important, the benefits of combining these designs. In the context of this review, the main point is that few studies are grounded in rigorous evidence, and many modeling studies present potential effects that are conditional on a long series of unproven assumptions rather than valid empirical evidence.

Not surprisingly, the vast majority of studies follow methodological practices developed for the economic evaluation of healthcare interventions. This strategy is endorsed by some scholars. ${ }^{14}$ Cost-utility analysis is the preferred type of analysis, and the focus of cost analyses is on healthcare resources. The formidable development of economic evaluation methods for healthcare programs and health technology assessment in general provides a strong methodological basis for the economic evaluation of nutritional interventions. Nevertheless, a thorough understanding of the consequences of the dissimilarities between traditional health technologies and nutrition interventions is fundamental to determine the potential of the dominant methods in health economics. Here, four issues that deserve further attention are addressed.

First, nutrition habits are culturally and socially embedded and therefore differ substantially from medical services. From one perspective, this situation calls for adequate empirical research to evaluate how to induce behavioral changes rather than simply investigating the comparative benefits of nutrients. Policymakers must know not only what nutrients should be promoted or discouraged but also how to trigger change. Both types of evidence are required to provide guidance for policymaking. From an economic perspective, one of the main issues is that nutrition habits are linked to eating and have wider implications for personal well-being. It is limiting to understand nutrition as only functional to health because people enjoy food and the rituals associated with it. This issue challenges the use of QALYs and other health-related measures to fully quantify the effects of nutritional interventions on well-being. Moreover, cost-benefit analyses of nutrition interventions should be expanded on a population basis to identify the most effective and economically sustainable strategies of action. ${ }^{57}$

Second, institutional guidelines, such as those of the National Institute for Health and Care Excellence (NICE) in the United Kingdom, the Agency for Drugs and Technologies in Health (CADTH) in Canada, or the Pharmaceuticals Benefits Advisory Committee (PBAC) in Australia, restrict the analysis of costs to those borne by the healthcare system and, in some circumstances, by the formal social care sector. Typically, they assume that efficiency improvement derives from a better allocation of resources pursued through the systematic use of costeffectiveness ratios. Given an amount of resources available (e.g., the budget of the public healthcare system), funding the most cost-effective interventions should allow for the maximization of health benefits. The issue with nutrition interventions is that food expenses are generally private and are not part of a public healthcare budget; thus, nutrition interventions aim to change consumer behavior. The relevant constraint in this case is the aggregate disposable family income, which can be used for all possible sources of well-being. For these reasons, research should investigate whether cost-effectiveness and cost-utility analyses are appropriate to evaluate the entire set of relevant costs and benefits associated with nutritional interventions. 
A third issue concerns distributional effects. Because food expenses are typically private, changes in food consumption patterns modify households' financial constraints and their allocation of resources. If, as is frequently the case, healthier diets induce variations in expenses, policies will have different economic implications across socioeconomic groups. For example, wealthier households have lower opportunity costs for additional food expenses than poorer households. In other words, unless they are highly subsidized, interventions targeted to change nutritional habits have relevant equity implications that cannot be addressed by standard economic evaluations developed in healthcare. Given the importance of private resources in nutrition decisions, analysis of the equity implications of different policies is much more relevant than in standard health technology assessment, in which the main issue is the best use of limited public resources and in which equity concerns involve the distribution of health effects across population groups rather than the distribution of costs.

A fourth issue involves the economic consequences of food policies. Agriculture and the food industry are strategic sectors in most countries, and agricultural products are traded worldwide. Health policies targeting food can substantially shift patterns of food consumption. Higher demand for healthier food can boost domestic production or imports, which may produce significant economic impacts (e.g., trade levels, prices of food) between and within nations. On the other hand, banning or limiting the consumption of suspected unhealthy food may have serious consequences for producers. Lock et al..$^{58}$ used a general equilibrium model to show that the benefits and costs of healthy diets may vary considerably between different populations because of the indirect effects of policies on agricultural production and trade. Policymakers frequently face broad trade-offs when establishing policies and should be provided with adequate economic evidence that encompasses all the costs and benefits of different courses of action. This issue calls for advances in research toward more holistic assessments that consider the potential health, trade, agricultural, and environmental implications of changes in diet. ${ }^{11,58}$

\section{CONCLUSION}

This review reveals that the four above-mentioned issues are virtually absent in the published literature. The few available studies tended to ignore the peculiarities of interventions aimed at healthier diets and, thus, risk providing weak economic evidence for policy. Both the paucity of published studies and the absence of specific accounts of the consequences of these nutrition policies for the economy at large suggest that much more inno- vative research is seriously required. Food consumption is a modifiable risk factor for a number of diseases, including cardiovascular diseases, diabetes, and cancer. The potential of major health gains from adequate food policies is high, but in its present state, the overall social evidence of specific interventions in this area is limited because of the paucity of studies and major methodological inadequacies.

\section{Acknowledgments}

The authors thank Prof. Mike Drummond for useful comments on an earlier version of the article; the user disclaimers apply.

Funding. Soremartec Italia (Ferrero Group; Alba, Italy) provided an unrestricted grant to conduct the research presented in the article. No information used in preparation of this manuscript was owned by the sponsor.

\section{REFERENCES}

1. World Health Organization. Global status report on non-communicable diseases 2010. Description of the global burden of NCDs, their risk factors and determinants. Available at: http://www.who.int/nmh/publications/ ncd_report2010/en/. Published April 2011. Accessed June 20, 2013.

2. Organisation for Economic Co-operation and Development. Obesity update 2012. Available at: http://www.oecd.org/health/49716427.pdf. Published 2012. Accessed June 20, 2013.

3. World Health Organization. Global strategy on diet, physical activity and health. Diet and physical activity: a public health priority. Available at: http:// www.who.int/dietphysicalactivity/strategy/eb11344/strategy_english_web.pdf. Published 2006. Accessed June 19, 2013.

4. World Health Organization. Global Health Observatory (GHO). Obesity: situation and trends. Available at: http://www.who.int/gho/ncd/risk_factors/ obesity_text/en/. Published 2008. Accessed May 21, 2013.

5. Mazzocchi M, Traill BW, Shogren JF. Fat Economics: Nutrition, Health, and Economic Policies. Oxford: Oxford University Press; 2009.

6. Sassi F. Obesity and the Economics of Prevention: Fit Not Fat. Paris: OECD Publishing; 2009.

7. Wong JB, Coates PM, Russell RM, et al. Economic analysis of nutrition interventions for chronic disease prevention: methods, research and policy. Nutr Rev. 2011;69:533-549.

8. Capacci S, Mazzochi M, Shankar B, et al. Policies to promote healthy eating in Europe: a structures review of policies and their effectiveness. Nutr Rev. 2012;70:188-200.

9. Gyles CL, Lenoir-Wijnkoop I, Carlberg JG, et al. Health economics and nutrition: a review of published evidence. Nutr Rev. 2012;70:693-708.

10. Nuijetn M, Lenoir-Wijnkoop I. Nutrition economics: an innovative approach to informed public health management. Eur J Pharmacol. 2011;668(Suppl 1):S133S137.

11. Lenoir-Wijnkoop I, Dapoigny M, Dubois D, et al. Nutrition economics - characterizing the economic and health impact of nutrition. Br J Nutr. 2011;105:157166.

12. Drummond M, Sculpher MJ, Torrance JW, et al. Methods for the Economic Evaluation of Health Care Programmes, $3^{\text {rd }}$ ed. Oxford: Oxford University Press; 2005.

13. Weatherly H, Drummond M, Claxton K, et al. Methods for assessing the costeffectiveness of public health interventions: key challenges and recommendations. Health Policy. 2009;93:85-92.

14. Kenkel DS, Manning W. Economic evaluation of nutrition policy. Or, there's no such thing as a free lunch. Food Policy. 1999;24:145-162.

15. Bray GA, Popkin BM. Dietary fat intake does affect obesity! Am J Clin Nutr. 1998;68:1157-1173.

16. Gold MR, Siegel JE, Russell LB, et al. Cost-Effectiveness in Health and Medicine. Oxford: Oxford University Press; 1996.

17. Moher D, Liberati A, Tetzlaff J, et al. Preferred reporting items for the systematic reviews and meta-analysis: the PRISMA statement. BMJ 2009;339:b2535. doi: 10.1136/bmj.b2535. 
18. Evers $\mathrm{S}$, Goossens $\mathrm{M}$, de Vet $\mathrm{H}$, et al. Criteria list for assessment of methodological quality of economic evaluations: consensus on health economic criteria. Int J Technol Assess Health Care. 2005;21:240-245.

19. Ackermann RY, Marrero DG, Hicks KA, et al. An evaluation of cost sharing to finance a diet and physical activity intervention to prevent diabetes. Diabetes Care. 2006;29:1237-1241.

20. Bibbins-Domingo K, Chertow GM, Coxson PG, et al. Reductions in cardiovascular disease projected from modest reductions in dietary salt. $\mathrm{N}$ Engl J Med. 2010;362:590-599.

21. Bós AM, Howard BV, Beresford SAA, et al. Cost-effectiveness analysis of a low-fat diet in the prevention of breast and ovarian cancer. J Am Diet Assoc. 2011;111:56-66.

22. Brannon SD, Tershakovec AM, Shannon BM. The cost-effectiveness of alternative methods of nutrition education for hypercholesterolemic children. Am J Public Health. 1997:87:1967-1970.

23. Burney J, Haughton B. EFNEP: a nutrition education program that demonstrates cost-benefit. J Am Diet Assoc. 2002;102:39-45.

24. Cobiac L, Vos T, Veerman JL. Cost-effectiveness of interventions to reduce dietary salt intake. Heart. 2010;96:1920-1925.

25. Cox RH, White AH, Gaylord CK. A video lesson series is effective in changing the dietary intakes and food-related behaviours of low-income homemakers. J Am Diet Assoc. 2003;103:1488-1493

26. Dall T, Fulgoni VL, Zhang $Y$, et al. Potential health benefits and medical cost savings from calorie, sodium, and saturated fat reductions in the American diet. Am J Health Promot. 2009;23:412-422.

27. Dallongeville J, Dauchet $\mathrm{L}$, de Mouzon $\mathrm{O}$, et al. Increasing fruit and vegetable consumption: a cost-effectiveness analysis of public policies. Eur J Public Health. 2010;21:69-73.

28. Dalziel K, Segal L, de Lorgeril M. A Mediterranean diet is cost-effective in patients with previous myocardial infarction. J Nutr. 2006;136:1879-1885.

29. Dollahite J, Kenkel D, Thompson CS. An economic evaluation of the Expanded Food and Nutrition Education Program. J Nutr Educ Behav. 2008;40:134-143.

30. Forster $\mathrm{M}$, Veerman JL, Barendregt JJ, et al. Cost-effectiveness of diet and exercise interventions to reduce overweight and obesity. Int J Obes. 2011;35:1071-1078.

31. Gans KM, Burkholder GJ, Risica PM, et al. Cost-effectiveness of minimal contact education strategies for cholesterol change. Ethn Dis. 2006;16:443-451.

32. Graves N, Barnett AG, Halton KA, et al. Cost-effectiveness of a telephonedelivered intervention for physical activity and diet. PLoS ONE 2009;4:e7135. doi: 10.1371/journal.pone.0007135.

33. Gray R, Malla S, Perlich K. Economic impacts of proposed limits of trans fats in Canada. Curr Agric Food Resour Issues 2006;7:149-161.

34. Gundgaard J, Nielsen JN, Olsen J, et al. Increased intake of fruit and vegetables: estimation of impact in terms of life expectancy and healthcare costs. Public Health Nutr. 2003;6:25-30.

35. Ha DA, Chisholm D. Cost-effectiveness analysis of interventions to prevent cardiovascular disease in Vietnam. Health Policy Plan. 2011;26:210-222.

36. Johannesson M, Fagerberg B. A health-economic comparison of diet and drug treatment in obese men with mild hypertension. J Hypertens. 1992;10:10631070 .

37. Joy $A B$, Pradhan V, Goldman GE. Cost-benefit analysis conducted for nutrition education in California. Calif Agric. 2006;60:185-191.

38. Magnus A, Haby MM, Carter $\mathrm{R}$, et al. The cost-effectiveness of removing television advertising of high-fat and/or high-sugar food and beverages to Australian children. Int J Obes. 2009;33:1094-1102.

39. Martikainen JA, Soini EJO, Laaksonen DE, et al. Health economic consequences of reducing salt intake and replacing saturated fat with polyunsaturated fat in the adult Finnish population: estimated based on the FINRISK and FINDIET studies. Eur J Clin Nutr. 2011;65:1148-1155.
40. Miners A, Harris J, Felix L, et al. An economic evaluation of adaptive e-learning devices to promote weight loss via dietary change for people with obesity. BMC Health Serv Res. 2012;12:190. doi: 10.1186/1472-6963-12-190.

41. Oster $G$, Thompson D. Estimated effects of reducing dietary saturated fat intake on the incidence and costs of coronary heart disease in the United States. J Am Diet Assoc. 1996;96:127-131.

42. Panagiotakos D, Sitara M, Pitsavos C, et al. Estimating the 10 -year risk of cardiovascular disease and its economic consequences, by the level of adherence to the Mediterranean Diet: the ATTICA study. J Med Food. 2007;10:239-243.

43. Rajgopal R, Cox RH, Lambur M, et al. Cost-benefit analysis indicates the positive economic benefits of the Expanded Food and Nutrition Education Program related to chronic disease prevention. J Nutr Educ Behav. 2002;34:26-37.

44. Robroek SJW, Polinder S, Bredt FJ, et al. Cost-effectiveness of a long-term Internet-delivered worksite health promotion programme on physical activity and nutrition: a cluster randomized controlled trial. Health Educ Res. 2012:27:399-410.

45. Sacks G, Veerman JL, Moodie M, et al. "Traffic-light" nutrition labelling and "junk food" tax: a modelled comparison of cost-effectiveness for obesity prevention Int J Obes (Lond). 2011;35:1001-1009.

46. Schuster E, Zimmerman ZL, Engle M, et al. Investing on Oregon's Expanded Food and Nutrition Education Program (EFNEP): documenting costs and benefits. $J$ Nutr Educ Behav. 2003;35:200-206.

47. Sevick MA, Miller GD, Loeser RF, et al. Cost-effectiveness of exercise and diet in overweight and obese adults with knee osteoarthritis. Med Sci Sports Exerc 2009;41:1167-1174.

48. Smith-Spangler CM, Juusola JL, Enns EA, et al. Population strategies to decrease sodium intake and the burden of cardiovascular disease: a cost-effectiveness analysis. Ann Intern Med. 2010;152:481-487.

49. te Velde SJ, Veerman JL, Tak NI, et al. Modelling the long term health outcomes and cost-effectiveness of two interventions promoting fruit and vegetable intake among schoolchildren. Econ Hum Biol. 2011:9:14-22.

50. Tsai AG, Glick HA, Shera D, et al. Cost-effectiveness of a low-carbohydrate diet and a standard diet in severe obesity. Obes Res. 2005;13:1834-1840.

51. van Baal PHM, van den Berg M, Hoogenveen RT, et al. Cost-effectiveness of a low-calorie diet and orlistat for obese persons: modelling long-term health gains through prevention of obesity-related chronic diseases. Value Health 2008;11:1033-1040.

52. van Keulen HM, Bosmans JE, van Tulder MW, et al. Cost-effectiveness of tailored print communication, telephone motivational interviewing, and a combination of the two: results of an economic evaluation alongside the Vitalum randomized controlled trial. Int J Behav Nutr Phys Act. 2010;7:64. doi: 10.1186/1479-5868-764

53. van Wier MF, Dekkers JC, Bosmans JE, et al. Economic evaluation of a weight control program with e-mail and telephone counseling among overweight employees: a randomized controlled trial. Int J Behav Nutr Phys Act. 2012 9:1-12.

54. Wootan MG, Reger-Nash B, Booth-Butterfield S, et al. The cost-effectiveness of $1 \%$ Or Less media campaigns promoting low-fat milk consumption. Prev Chronic Dis. 2005;2:1-10.

55. World Health Organization. World Health Report 2000: Health Systems: Improving Performance. Geneva: WHO; 2000.

56. Hooper $\mathrm{L}$, Abdelhamid A, Moore $\mathrm{HJ}$, et al. Effect of reducing total fat intake on body weight: systematic review and meta-analysis of randomized controlled trials and cohort studies. BMJ 2012;345:e7666. doi: 10.1136/bmj.e7666.

57. Agostoni C, Fattore G. Growth outcome: nutritionist perspective. World Rev Nutr Diet. 2013;106:12-18

58. Lock K, Smith RD, Dangour AD, et al. Health, agriculture, and economic effects of adoption of healthy diet recommendations. Lancet 2010;376:1699-1709. 